\title{
Usefulness of Unilateral Nasal Packing for Recurred Septal Deviation within a Month after Septoplasty: Preliminary Study
}

\author{
Yoon Hee Lee, Jin Soon Chang, $\mathrm{MD}^{2}$, Joo Hyun Jung, $\mathrm{MD}^{3}$, Seon Tae Kim, $\mathrm{MD}^{3}$ and $\mathrm{Il}$ Gyu Kang, $\mathrm{MD}^{3}$ \\ ${ }^{\text {I} S t u d e n t, ~ G a c h o n ~ U n i v e r s i t y ~ S c h o o l ~ o f ~ M e d i c i n e, ~ I n c h e o n, ~ K o r e a ~}$ \\ ${ }^{2}$ Department of Otolaryngology-Head and Neck Surgery, Inje University Seoul Paik Hospital, Seoul, Korea \\ ${ }^{3}$ Department of Otolaryngology-Head \& Neck Surgery, Medical Faculty, Gil Medical Center, Gachon University, Incheon, Korea
}

\begin{abstract}
Background and Objectives: To investigate the effect of unilateral nasal packing on the correction of recurred septal deviation after septoplasty.

Materials and Method: We analyzed 12 patients who had undergone septoplasty and developed septal deviation recurrence. Polyvinylacetate and Vaseline gauze were inserted into the nasal passage on the convex side of the septum for 4 days in order to shift the septum to the midline. We analyzed nasal symptoms, acoustic rhinometric results, and endoscopic findings before and after unilateral packing in order to evaluate the treatment outcomes.

Results: Ten (83\%) out of 12 patients showed improvements in nasal obstruction, acoustic rhinometric results, and endoscopic findings. The mean visual analogue scale (VAS) score for nasal obstruction was $5.25 \pm 1.60$ before and $2.08 \pm 1.50$ after packing $(\mathrm{p}=0.004)$. The minimal cross-sectional area (MCA) improved from $0.17 \pm 0.14$ to $0.27 \pm 0.13(\mathrm{p}=0.002)$, and the mean endoscopic score improved from $2.0 \pm 0.43$ to $1.08 \pm 0.29$ ( $\mathrm{p}=0.002$ ).

Conclusion: Unilateral nasal packing was a safe, easy, and effective method for correcting recurred septal deviation after septoplasty.
\end{abstract}

KEY WORDS: Septal deviation · Packing · Acoustic rhinometry.

\section{INTRODUCTION}

Septoplasty is one of the most common procedures for treating septal deviation. However, there is a discrepancy between the opinion that septoplasty is an easy operation, and the relatively high failure and complication rates associated with this procedure. The success rates for septoplasty reported in the literature range from 43 to $85 \%{ }^{1-4)}$ and vary depending upon the tool used to measure the surgical outcomes. There are few treatment options for correcting recurrent septal deviation after septoplasty aside from medical treatment or revision septoplasty. Moreover, revision septoplasty is difficult to perform because mucosal re-dissection is more difficult and the complication rate is higher than that of primary septoplasty. ${ }^{5)}$ Thus, recurrent septal deviation after septoplasty is a significant concern for surgeons. We hypothesized that since the bony-cartilageous junction is partially detached in patients with recurred septal deviation, unilateral nasal packing on the convex side could shift the septum to the midline. Herein, we investigated the effect of repeated unilateral nasal packing to correct recurred septal deviation soon after septoplasty.

\section{SUBJECTS AND METHODS}

Patients

This study was approved by the Institutional Review Board of Gachon University Gil Medical Center (Incheon

Received: February 17, 2018 / Revised: April 6, 2018 / Accepted: May 23, 2018

Address for correspondence: II Gyu Kang, MD, Department of Otolaryngology-Head and Neck Surgery, Gil Medical Center, 21 Namdong-daero 774beon-gil, Namdong-gu, Incheon 21565, Korea

Tel: +82-32-460-3764, Fax: +82-32-467-9044, E-mail: eyik@naver.com 
City, South Korea). Patients provided informed consent after receiving a complete description of the study protocol. From March 2011 to March 2012, a prospective, uncontrolled clinical study was conducted at a teaching hospital. Twelve patients were enrolled, who had undergone septoplasty and developed recurrent septal deviation. The inclusion criteria for the patients were as follows: 1) age greater than 18 years, 2) a postoperative period less than 1 month, 3 ) individuals who developed a nasal obstruction on the convex side, and 4) patients in which the septum was corrected well after septoplasty. The exclusion criteria were 1) nasal obstruction due to inferior turbinate hypertrophy, nasal polyps, concha bullosa, or allergic rhinitis, 2) patients with severe asthma, and 3 ) age greater than 65 years.

\section{Surgical technique}

We performed the initial septoplasty using the following protocol. With the patient under general or local anesthesia, a caudal incision (hemitransfixion incision) was made and flaps were elevated through this incision. After subperichondrial dissection, complete release of the junction between the cartilaginous septum and maxillary crest was achieved as well as partial release of the septal cartilage from the vomer and perpendicular plate of the ethmoid. ${ }^{6}$ Next, the deviated bone and cartilage were resected $(1.5 \times$ $1.5 \mathrm{~cm}$ sized L-strut ${ }^{7)}$ was remained) after which it was repositioned and inserted into the site of removal. When the cartilage deviated after separation of the bone-cartilage junction, we incised the deviated septal cartilage and manipulate the cartilage and inserted it, or made crosshatch incisions on the concave side of septum. After ensuring that the septum was straight, the incision site was sutured and bilateral nasal cavity was packed with polyvinylacetate (PA, Merocel $^{\circledR}$; Medtronic Xomed, Jacksonville, FL, USA) for 2 days. At the same time, bilateral inferior turbinate mucosal resection was performed.

\section{Interventions}

Unilateral nasal packing was performed immediately after finding recurrence of nasal septal deviation. Before unilateral nasal packing, we first removed nasal secretions and took endoscopic photos, checked the questionnaire, and performed acoustic rhinometry. After these procedures, we packed the nasal passage with Merocel ${ }^{\circledR}$ and one or two pieces of Vaseline gauze on the convex side to shift the septum to the midline (Fig. 1). Two days later, we removed the packing materials, and repacked with PA and Vaseline gauze for 2 more days to prevent infection. While the nasal passage was packed, we prescribed antibiotics to prevent infection.

\section{Outcome measurements}

We measured the degree of nasal obstruction using a visual analogue scale (VAS) of 0 (no nasal obstruction) to 10 (complete nasal obstruction). Endoscopic findings were rated on a scale of 0 to 3 ( 0 : normal septum, 1: mild deviation is defined as less than half of the nasal floor width, 2: moderate deviation is defined as half of the nasal floor width and 3: severe deviation is defined as more than half of the nasal floor width) by 2 otolaryngologists (I.G.K and S.T.K) before and after nasal packing removal. We checked the intra-nasal photos (Fig. 2), VAS, and acoustic rhinometric results before and on the last follow-up day after removing nasal packing to evaluate the ability of unilateral packing to correct recurred septal deviation. Acoustic rhinometric measurements were performed using the RhinoMetrics SRE 2000 (Interacoustics AS, Assens, Denmark).

\section{Statistical analysis}

Categorical variables were analyzed with the Wilcoxon signed rank test using the Statistical Package for the Social Sciences (SPSS), version 18.0 (SPSS Inc., Chicago, IL, USA). $\mathrm{P}$-values $<0.05$ were considered statistically significant.

\section{RESULTS}

Out of the 12 patients, 8 were male and 4 were female. The mean age was $33.3 \pm 13.0$ years. The mean follow-up period was $3.3 \pm 2.4$ months. All patients showed recurred septal deviation in bony-cartilageous junction area (between septal catilage and perpendicular plate of ethmoid, vomer) and no caudal septal deviation or high septal deviation only. Ten (83\%) out of 12 patients showed improved nasal obstruction as well as endoscopic finding scores and increased minimal cross-sectional area (MCA). The mean VAS scores for nasal obstruction were $5.25 \pm 1.60$ before and $2.08 \pm$ 1.50 after nasal packing $(\mathrm{p}=0.004)$. The mean endoscopic scores also improved from $2.0 \pm 0.43$ to $1.08 \pm 0.29$ ( $p=0.02$; Table 1). The mean MCA was $0.17 \pm 0.14 \mathrm{~cm}^{2}$ before and 0.27 $\pm 0.13 \mathrm{~cm}^{2}$ after nasal packing $(\mathrm{p}=0.002)$. No significant complications were observed during or after nasal packing. 


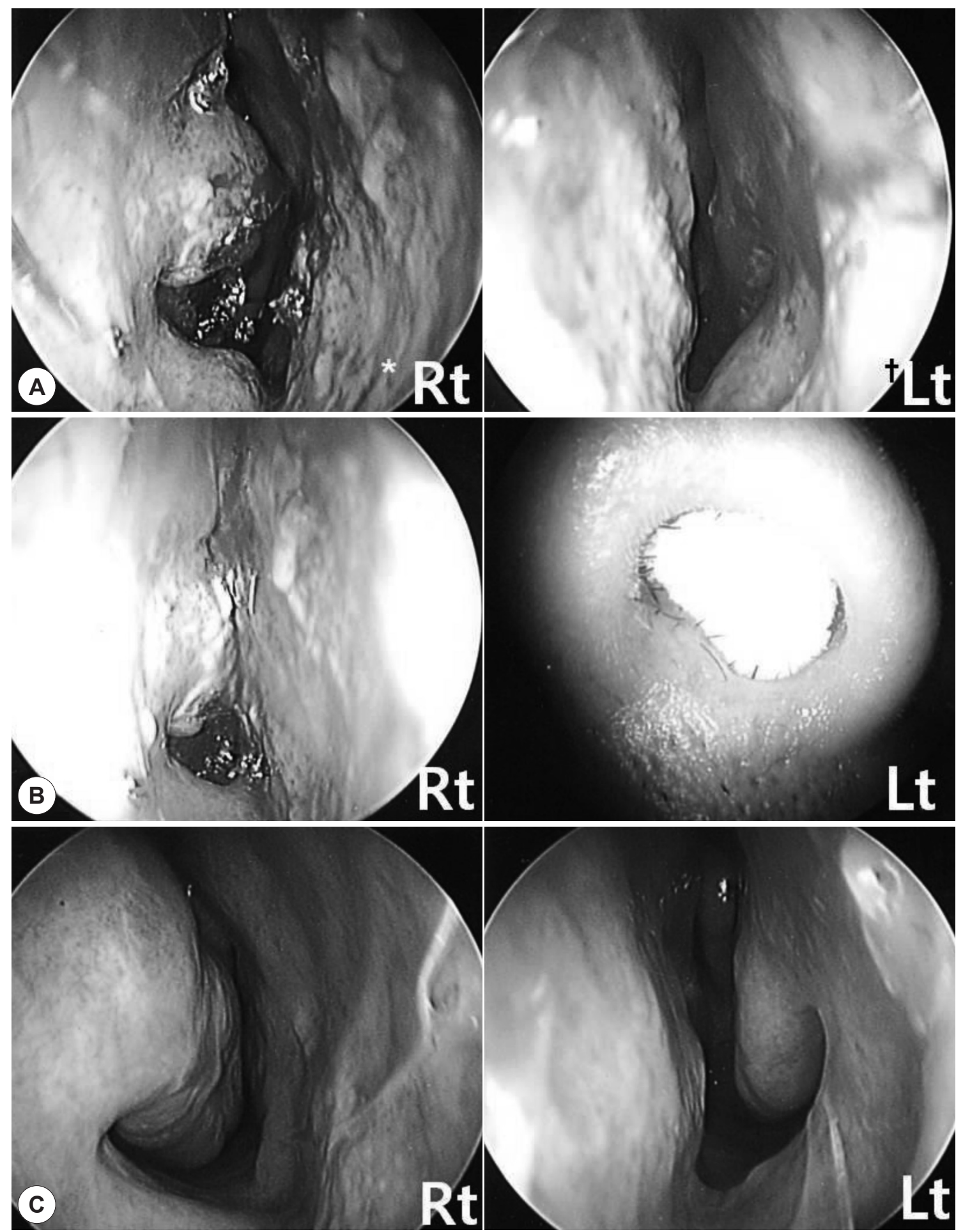

Fig. 1. These pictures show recurrent septal deviation to the left side 7 days after septoplasty (A). We packed the left nasal cavity with polyvinyl acetate and Vaseline gauze to shift the deviated septum to the midline (B). The corrected deviated septum 9 months after nasal packing (C). The minimal cross-sectional area (MCA) increased from 0.04 to $0.12 \mathrm{~cm}^{2}$. Rt: right, Lt: left.

\section{DISCUSSION}

Nasal septum deviation is a very common cause of nasal obstruction and corrected by septoplasty. However, Dinis et al. ${ }^{8)}$ reported that only $42 \%$ of patients showed good to excellent results while the majority of these individuals 


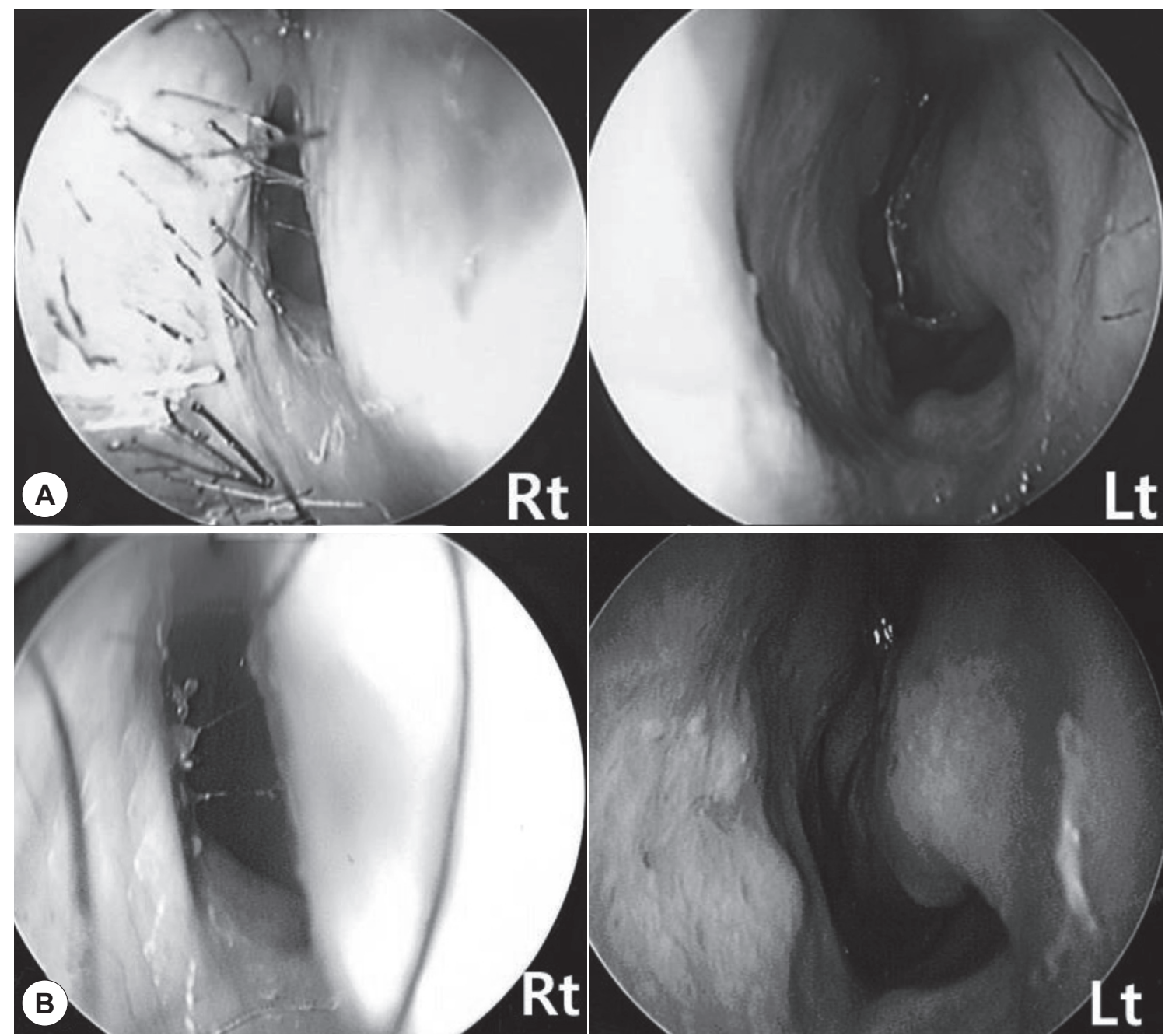

Fig. 2. In this patient, right-side recurrent septal deviation was visible (A). 4 months after unilateral nasal packing, the deviation was corrected $(B)$

Table 1. Patient demographic data, visual analogue scale of nasal obstruction, endoscopic findings, and acoustic rhinometric results before and after unilateral nasal packing

\begin{tabular}{|c|c|c|c|c|c|}
\hline & $\begin{array}{l}\text { Patient gender/ } \\
\text { age }\end{array}$ & $\begin{array}{c}\text { VAS of NO } \\
\text { (pre- } \rightarrow \text { post-packing) }\end{array}$ & $\begin{array}{l}\text { Endoscopic findings } \\
\text { (pre- } \rightarrow \text { post-packing) }\end{array}$ & $\begin{array}{l}\text { Follow-up period } \\
\text { (months) }\end{array}$ & $\begin{array}{c}\text { MCA }\left(\mathrm{cm}^{2}\right) \\
(\text { pre- } \rightarrow \text { post-packing })\end{array}$ \\
\hline 1. & $M / 29$ & $4 \rightarrow 2$ & Improved $(2 \rightarrow 1)$ & 2 & $0.13 \rightarrow 0.20$ \\
\hline 2. & $\mathrm{~F} / 46$ & $4 \rightarrow 1$ & Improved $(2 \rightarrow 1)$ & 2 & $0.25 \rightarrow 0.47$ \\
\hline 3. & $M / 26$ & $5 \rightarrow 5$ & Not improved $(2 \rightarrow 2)$ & 1.5 & $0.18 \rightarrow 0.19$ \\
\hline 4. & $M / 53$ & $7 \rightarrow 2$ & Improved $(2 \rightarrow 1)$ & 3 & $0.04 \rightarrow 0.14$ \\
\hline 5. & $M / 30$ & $5 \rightarrow 1$ & Improved $(2 \rightarrow 1)$ & 3.5 & $0.11 \rightarrow 0.27$ \\
\hline 6. & $M / 48$ & $8 \rightarrow 2$ & Improved $(3 \rightarrow 1)$ & 9 & $0.04 \rightarrow 0.12$ \\
\hline 7. & $\mathrm{~F} / 22$ & $5 \rightarrow 1$ & Improved $(2 \rightarrow 1)$ & 3 & $0.04 \rightarrow 0.26$ \\
\hline 8. & $M / 23$ & $4 \rightarrow 5$ & Not improved $(2 \rightarrow 2)$ & 5 & $0.33 \rightarrow 0.34$ \\
\hline 9. & $M / 53$ & $4 \rightarrow 1$ & Improved $(2 \rightarrow 1)$ & 2.5 & $0.1 \rightarrow 0.2$ \\
\hline 10. & $F / 22$ & $7 \rightarrow 1$ & Improved $(2 \rightarrow 1)$ & 1 & $0.26 \rightarrow 0.30$ \\
\hline 11. & $M / 29$ & $3 \rightarrow 1$ & Improved $(2 \rightarrow 1)$ & 1 & $0.48 \rightarrow 0.56$ \\
\hline 12. & $F / 18$ & $7 \rightarrow 3$ & Improved $(2 \rightarrow 1)$ & 6.5 & $0.09 \rightarrow 0.20$ \\
\hline
\end{tabular}

Endoscopic findings were scored as 1: mild septal deviation, 2: moderate septal deviation, and 3: severe septal deviation. VAS: visual analogue scale (0: no nasal obstruction, 10: complete nasal obstruction), NO: nasal obstruction, MCA: minimal cross-section area 
achieved either slightly to moderately successful outcomes $(35 \%)$ or poor to mediocre results $(23 \%)$ after septoplasty. However, revision septoplasty is not easy to perform and revision surgery may also not be effective, especially when there are residual anatomical abnormalities, unless concomitant medical therapy is optimized and administered. ${ }^{5)} \mathrm{We}$ postulated that unilateral nasal packing could shift a recurred deviated septum to the midline because the junction between septal cartilage and surrounding bone is detached soon after septoplasty. In the current study, we used PA and Vaseline gauze. These packing materials are the most commonly used after nasal surgery, and PA is able to expand lengthwise. In a previous investigation, we found that PA prevents frontal sinus ostium stenosis when placed in the frontal sinus ostium after functional endoscopic sinus surgery because PA has an expansile effect. ${ }^{9)}$ Many techniques, including suturing, ${ }^{10)}$ scoring, and repositioning, ${ }^{1112)}$ have been developed to change the shape of curved cartilage. Sometimes these techniques produce permanent results and other times the cartilage regains its original curvature over time. One possible cause of recurrent septal deviation after septoplasty is simple incomplete resection of the cartilaginous or bony septum that was not obvious at the time of surgery. ${ }^{5)}$ Two patients in the current study did not show any improvement after unilateral nasal packing. In these individuals, the septum was not easily moved when the nasal passage was packed with PA compared to the other patients. That meant that the complete release of the junction between the cartilaginous septum and surrounding structures was not achieved by the packing. In addition, these 2 patients showed recurrent septal deviation within 7 days after unilateral packing removal. Although follow up period of this study is short, the patients that had shown improved septal deviation after unilateral nasal packing did not show recurred septal deviation 1 month later after packing removal. We believe that unilateral nasal packing not only affects the septal cartilage curvature, but also shifts partially separated cartilage to the mildline soon after septoplasty. Before unilateral nasal packing, surgeons must determine whether the septum is still causing nasal obstruction. The physician must be certain that there are no other causes of obstruction such as turbinate hypertrophy, concha bullosa, nasal valve deformity, or polyps. While packing the nasal passage, we did not observe any major complications. Additionally, minor complications such as nasal obstruction, mild headache, and rhinorrhea subsided after packing removal.

\section{CONCLUSION}

Our findings indicate that unilateral nasal packing is a relatively safe, simple, and conservative technique. We therefore recommend that unilateral nasal packing should be performed to correct recurred septal deviation after septoplasty before any other procedures are attempted.

\section{REFERENCES}

1) Siegel NS, Gliklich RE, Taghizadeh F, and Chang Y. Outcomes of septoplasty. Otolaryngol Head Neck Surg 2000;122:228-32.

2) Thomas JN. S.M.R. - a two-year follow-up survey. J LaryngolOtol 1978;92:661-6.

3) Illum P. Septoplasty and compensatory inferior turbinate hypertrophy: Long-term results after randomized turbinoplasty. Eur Arch Otorhinolaryngol 1997;254(suppl 1):S89-92.

4) Fjermedal O, Saunte C, Pederson S. Septoplasty and/or submucous resection? 5 years nasal septum operations. J Laryngol Otol 1988; 102:796-8.

5) Sillers MJ, Cox AJ 3rd, Kulbersh B. Revision septoplasty. Otolaryngol Clin North Am 2009;42(2):261-78.

6) Kim YD. Septoplasty and Turbinoplasty; Current Concept and Technique. J Rhinol 2012;19(1):19-28.

7) Jin HR, Won TB. Septoplasty; Current Concept and Technique. Septoplasty. J Rhinol 2008;15(1):13-29.

8) Dinis PB, Haider H. Septoplasty: Long term evaluation of results. Am J Otolaryngol 2002;23:85-90.

9) Kang IG, Jung JH, Woo JH, Cha HE, Kim ST. The effect of expandable polyvinyl acetate packing for preventing stenosis of the frontal sinus ostium Am J Rhinol Allergy 2010;24(5):392-5.

10) Kang JM, Nam ME, Dhong HJ, Kim HY, Chung SK, Kim JH. Modified mattress suturing technique for correcting the septal high dorsal deviation around the keystone area. Am J Rhinol Allergy 2012; 26(3):227-32.

11) Lawson $W$, Westreich R. Correction of caudal deflections of the nasal septum with a modified Goldman septoplasty technique: how we do it. Ear Nose Throat J 2007;86(10):617-20.

12) Yang JW, Kim SI, Kwon JW, Park DJ. Are Cross-hatching Incisions Mandatory for Correction of Cartilaginous Septal Deviation? Clin Exp Otorhinolaryngol 2008;1(1):20-3. 